\title{
DIFFICULT AND RECURRENT MENINGITIS
}

L Ginsberg

J Neurol Neurosurg Psychiatry 2004;75(Suppl I):i16-i21. doi: 10.1136/nip.2003.034272

hen can the management of meningitis become difficult? Broadly speaking, there are 1 three main potential areas of concern:

- Clinical diagnosis - The classical symptoms and signs of acute meningitis may be absent in the very young, the very old and the very sick. Chronic meningitis requires a degree of clinical suspicion, as again signs of meningism may be minimal or absent.

- Laboratory diagnosis_Difficulties may arise in patients whose cerebrospinal fluid (CSF) examination yields a typical "aseptic meningitis" picture (elevated white cell count, predominantly lymphocytic, no organisms on Gram stain, normal or elevated protein concentration, normal or reduced glucose concentration) because of the breadth of the differential diagnosis, both infective and non-infective (table 1). In these circumstances, it is particularly important to exclude or diagnose tuberculosis, cryptococcosis, and other fungal infections, partially treated pyogenic meningitis, neurosyphilis, and Lyme disease, because of the need for urgent treatment and the consequences of failure to treat.

- Treatment-Two vexed questions are the choice of antimicrobial drug in particular clinical settings, and the role of corticosteroid treatment.

In this article, the major causes of meningitis are considered in turn, highlighting the difficulties which may arise under these three headings. Finally, the syndrome of recurrent meningitis is discussed separately, as its causes and management may differ from those of an isolated attack of acute meningitis. Inevitably, there will be overlap between the content of this article and others in the present issue, but, whenever possible, the discussion here will be restricted to immunocompetent patients.

\section{PYOGENIC MENINGITIS}

Apart from the general difficulties of establishing a clinical diagnosis of pyogenic meningitis in certain patient groups, as listed previously, specific problems may arise in the case of meningococcal infection. Thus, in a recent publication from one of the medical defence organisations, ${ }^{1}$ there is the cautionary tale of a teenager presenting with headache and fever but no signs of meningism, who had meningococcal infection with predominant septicaemia rather than meningitis. Though the meninges were relatively spared (at least clinically) the disease was severe, diagnosis was delayed, and the patient suffered bilateral above-knee amputations, hypoadrenalism, and other complications. To compound the problem, the rash of meningococcal sepsis may initially be subtle and relatively localised. ${ }^{2}$ Like the rash, signs of meningism may evolve in minutes to hours, necessitating failsafe mechanisms for regular patient review and handover.

The laboratory diagnosis of untreated pyogenic meningitis is usually straightforward once CSF has been taken. The difficult issue here is whether the patient should undergo computed tomography (CT) of the brain before lumbar puncture. Recent evidence confirms the view that only a very small proportion of patients with suspected meningitis are at risk of brain herniation following lumbar puncture. ${ }^{3}$ Conversely, a normal CT head scan does not preclude herniation resulting from raised intracranial pressure caused by meningitis itself. Despite these observations, many physicians feel obliged to request a pre-puncture CT through fear of litigation. In such circumstances, there is a potential risk to the patient from delayed treatment. Certainly, a patient should undergo CT of the head before lumbar puncture in the presence of papilloedema, deteriorating level of consciousness, and/or focal neurological signs. In these cases, intravenous antibiotics should be started before the scan, after blood cultures have been taken (see case study l).

The treatment of pyogenic meningitis necessarily begins at a time when the antibiotic sensitivities of the causative organism are unknown. As a general principle, it is prudent for the neurologist to share management with a specialist in microbiology/infectious diseases. With the emergence of penicillin resistant strains of the meningococcus and pneumococcus, initial empirical treatment with a third generation cephalosporin (cefotaxime or ceftriaxone) is recommended. To this, vancomycin may be added if staphylococcal infection is suspected (that is, 
Case study 1

- A 70 year old man was brought to the accident and emergency department in status epilepticus. No antecedent history was available. He was febrile but there were no localising signs, as far as could be ascertained. Fundoscopy was not possible: one eye had been enucleated, the cornea was cloudy on the other side. During immediate resuscitation and control of seizures, blood cultures were taken and he was given high dose intravenous benzylpenicillin. A CT cranial scan did not show a mass lesion but there were small fluid (pus) levels in the lateral ventricles. At subsequent lumbar puncture, turbid CSF was obtained, containing 2000 white cells $/ \mu$ l (all polymorphs), protein concentration was $2.8 \mathrm{~g} / \mathrm{l}$, and glucose was undetectable. Though the sample proved sterile on culture, Gram positive cocci were seen on microscopy and fully sensitive Streptococcus pneumoniae was grown from the blood cultures. The patient received a 14 day course of high dose penicillin and made a complete recovery.

- Comment-This patient presented several years ago, before the polymerase chain reaction and other modern diagnostic techniques were available. Despite the negative CSF culture, presumably a consequence of the initial dose of antibiotics, it was still possible to confirm a diagnosis of pneumococcal meningitis from CSF microscopy and blood cultures. The good outcome is perhaps surprising, given the overall prognosis of pneumococcal meningitis and his condition at presentation. It may in part be explained by the speed and order of the initial management, antibiotics being given immediately rather than waiting for the CT scan and lumbar puncture.

in the context of head injury, neurosurgery or CSF shunt) or ampicillin for listeriosis (neonates, elderly, and immunocompromised patients). Vancomycin is also added in areas of the world where cephalosporin resistant pneumococci are encountered. ${ }^{4}$ Having said this, penicillin resistance is relatively rare in the UK, cephalosporin resistance thankfully not yet an issue, and if the organism is sensitive, benzylpenicillin remains the best drug. The author therefore prefers to treat patients with suspected meningococcal or pneumococcal meningitis initially with a combination of benzylpenicillin

Table 1 Causes of an "aseptic meningitis" cerebrospinal fluid picture*

- Partially treated bacterial meningitis
- Viral meningitis
- Tuberculous meningitis
- Spirochaetes: syphilis, Lyme disease, leptospirosis
- Other bacteria-for example, brucellosis, mycoplasma
- Cerebral malaria
- Fungal meningitis
- Parameningeal infection (spinal or intracranial abscess, venous sinus
thrombosis, occult paranasal sinus infection)
- Endocarditis
- Malignant meningitis (carcinoma, lymphoma, leukaemia)
- Subarachnoid haemorrhage
- Chemical meningitis (see text)
Sarcoidosis
- Other chronic inflammatory diseases (see text)
- Drugs
- Mollaret's meningitis (see text)

*With certain obvious exceptions (for example, malignant or tuberculous meningitis), many of these causes are potentially recurrent (see text). and a cephalosporin, provided there is no contraindication, dropping the cephalosporin once the organism's penicillin sensitivity is established.

At the risk of sounding old fashioned, a two hourly intravenous penicillin regimen is also recommended. ${ }^{5}$ Thus, patients initially receive a $2.4 \mathrm{~g}$ bolus of benzylpenicillin and then $1.2 \mathrm{~g}$ every two hours until there is evidence of clinical improvement (usually within 48-72 hours), at which point the regimen can be relaxed to four or six hourly, though with the same total daily dose $(14.4 \mathrm{~g})$. The reasoning behind this intensive approach is twofold. First, it may ensure a more constant high CSF antibiotic concentration. Second, and more important, it covers the risk of a dose being missed (pace the nursing staff). If a dose is inadvertently not given in a two hourly schedule, then the patient will have gone without antibiotics for four hours and no harm is likely to have been done. But if the patient is receiving penicillin four or six hourly and a dose is missed, he or she will have gone without the drug for eight or 12 hours, with potentially serious consequences in the context of pyogenic meningitis.

The role of corticosteroids in the immediate management of acute bacterial meningitis is becoming clearer. For some years, there has been evidence favouring treatment with dexamethasone during the first $2-4$ days to prevent audiological complications in children with Haemophilus influenzae meningitis (now rare following the introduction of the vaccine). For adults with pyogenic meningitis, a recent trial has shown that early treatment with dexamethasone improves outcome (morbidity and mortality) and does not increase the risk of gastrointestinal bleeding. ${ }^{6}$ Particularly encouraging results were seen in the subgroup with pneumococcal meningitis. But there are still theoretical and practical concerns about the blanket use of steroids in this setting. With the emergence of antibiotic resistant pneumococcal strains, the influence of corticosteroids on antibiotic penetration into the CSF must be considered. At least in experimental meningitis in animals, concomitant steroids reduce vancomycin penetration into CSF.

Another issue is the risk that adjunctive dexamethasone may mask clinical evidence of antibiotic failure, leading some authors to recommend a second lumbar puncture 2448 hours after onset to confirm microbiological improvement. ${ }^{4}$ Even in the modern era, pneumococcal meningitis remains a devastating illness. ${ }^{7}$ Antibiotic treatment alone is not enough. With the caveats above, adjuvant steroids are probably of limited benefit but the hope is for more specific anti-inflammatory agents in the future, directed, for example, against CSF cytokines, matrix metalloproteinases, and reactive oxygen species.

\section{TUBERCULOUS MENINGITIS}

Meningeal infection with Mycobacterium tuberculosis is the example par excellence of a form of meningitis where difficulties in diagnosis and management regularly occur. The fact remains that there is still no single diagnostic method which is both sufficiently rapid and sensitive. Inevitably, therefore, many patients will receive anti-tuberculous chemotherapy without a confirmed diagnosis. The physician must balance the risks of delaying treatment against those of major side effects of medication, notably hepatotoxicity.

The initial clinical and laboratory picture of tuberculous meningitis (TBM) is relatively non-specific, resembling many 
other causes of subacute meningitis. Typically, headache, fever, and meningism progress to coma within a few days (sometimes weeks), the CSF being lymphocytic with a low glucose concentration, though many atypical presentations have been described. The index of clinical suspicion will be raised in at-risk groups: ethnic minorities, immigrants, and the immunocompromised. Other predictors of TBM have recently been identified in an adult population in Vietnam: younger age, longer duration of illness, relatively lower blood white cell count and CSF total white cell count, and lower percentage of neutrophils in the CSF. ${ }^{8}$

Laboratory confirmation of TBM may be achieved by several means, each with its own drawbacks. The gold standard for many years was, and indeed still remains, the detection of tubercle bacilli in the CSF by microscopy and/or culture. Direct microscopic examination of the CSF, however, is notoriously sensitive to sample volume and time spent searching for acid-alcohol fast bacilli. Despite improvements in technology, culture takes too long for early diagnosis. Its value is in the subsequent confirmation of TBM in patients who have started anti-tuberculous chemotherapy solely on clinical grounds.

The false negative rate of the direct smear and the delay in obtaining culture results have prompted a search for alternative diagnostic tests based on modern molecular methods. One approach is to measure a surrogate marker such as CSF adenosine deaminase activity. The activity of this enzyme is raised in patients with TBM but it cannot discriminate tuberculosis reliably from important differential diagnoses including partially treated pyogenic meningitis. The detection of $M$ tuberculosis nucleic acid in CSF by the polymerase chain reaction (PCR) and allied techniques is more promising. Having said this, the sensitivity of PCR is probably no different from that of a carefully analysed smear. The advantage of PCR is in patients where CSF was taken after anti-tuberculous treatment had begun, as mycobacterial nucleic acid is likely still to be detectable, but the sensitivity of smear and culture will have fallen dramatically.

Other investigations used in patients with suspected TBM include the tuberculin skin test, but this is subject to both false positive and false negative results. The chest $x$ ray may show a miliary appearance in a small proportion of cases. Brain imaging by CT or magnetic resonance (MR) may reveal hydrocephalus or basal meningeal enhancement in TBM but the appearances are non-specific, being shared by other subacute and chronic meningitides. A significant proportion of patients with TBM will have normal imaging. Tuberculomas are seen only in a minority. Taken together with the imperative of early treatment to reduce the chance of a fatal outcome or severe complications, all these considerations mean that it is better generally to start treatment without delay in a patient with suspected TBM, diagnostic confirmation coming later.

The drug treatment of TBM continues to pose problems. Unlike pulmonary tuberculosis, clear guidelines about the choice of regimen and duration of treatment are lacking. By analogy with pulmonary disease, patients generally receive an intensive period of treatment then a continuation phase. In TBM, four anti-tuberculous drugs are given initially: isoniazid (with pyridoxine cover), rifampicin, pyrazinamide, and one other, usually ethambutol or streptomycin. Of these, isoniazid is likely to be the most important, given its ability to achieve high CSF concentrations and its bactericidal activity. Isoniazid and rifampicin are continued in the maintenance phase and pyrazinamide is also often used throughout the course. Though some reports suggest a six month total course of treatment is adequate for TBM, the author uses four drugs for three months followed by three drugs for another nine months.

Response is gradual-there may be no clinical improvement in the first two weeks-and even after one year of treatment, relapses may be seen. At that stage, it may be difficult to decide whether relapse is caused by ongoing infection or a paradoxical treatment reaction. Late expansion of a tuberculoma is a well recognised, albeit incompletely understood, immunological phenomenon. In such circumstances, continuing treatment with a combination of antituberculous drugs and high dose corticosteroids is empirical but unavoidable, until stabilisation and the beginning of resolution are seen. The use of adjunctive corticosteroids at the beginning of a course of anti-TBM treatment remains controversial. ${ }^{9}$ There is more convincing evidence of benefit in children. In adults, coma at presentation is an indication. The dose is typically dexamethasone $16 \mathrm{mg} /$ day (or an equivalent high dose of prednisolone) for the first 2-4 weeks of anti-TBM treatment, then tapering to zero over a similar period.

Other problems in the management of TBM include the challenge of multi-drug resistant disease, to which there is no easy answer. Theoretically, patients with multi-drug resistant TBM should be treated according to the drug resistance profile of the causative organism in each individual case. The patient, however, may well be moribund by the time such information becomes available, though the detection of mutations coding for specific drug resistance may permit more rapid characterisation of resistant disease.

The role of neurosurgery in the management of TBM is uncertain. If a patient on anti-TBM treatment suffers a deterioration in level of consciousness, or develops new physical signs, urgent re-imaging is needed, looking in particular for hydrocephalus and abscess formation, the presence of which will require a neurosurgical opinion.

\section{OTHER BACTERIA}

While pyogenic meningitis and TBM demand urgent management decisions, other bacteria may present more indolently and may be associated with a predominantly lymphocytic CSF. Potentially the most chronic meningitis occurs in syphilis, though the late manifestations are rarely seen nowadays. Another spirochaete, Borrelia burgdorferi, causes both acute and chronic neurological complications. Acute Lyme neuroborreliosis may be indicated clinically by the association of a lymphocytic meningitis with painful radiculopathy or cranial neuropathies, typically bilateral facial palsies. The diagnosis of meningitis caused by a third group of spirochaetes, leptospirosis, is usually aided by knowledge of the patient's occupation and by the presence of clinical features in other systems. Occupational risk may also lead to a diagnosis of brucellosis, which may present with an aseptic meningitis picture. Finally, mycoplasma infection can cause a lymphocytic meningitis, the diagnosis resting on serological tests (cold agglutinins and antimycoplasma antibodies) or the detection of the organism by PCR. 


\section{VIRAL MENINGITIS}

Viral infection is the most common cause of acute aseptic meningitis. Various viruses have been implicated (table 2). Very often, the causative organism is never identified, or obtaining serological proof of a particular viral infection is delayed, though PCR is improving the speed of microbiological diagnosis. The absence of information about a specific viral cause may lead to initial diagnostic confusion, given the range of alternatives (table 1). In particular, viral meningitis may present with sudden onset of headache and meningism, thereby mimicking subarachnoid haemorrhage. The treatment of viral meningitis is symptomatic, and the condition is self limiting in the great majority of cases.

\section{FUNGAL INFECTION}

Though cryptococcal meningitis is usually seen in patients with a defective immune system, it can occur in immunocompetent individuals. It presents as a subacute or chronic meningitis which resembles TBM. Other, more challenging, presentations have been described, even including isolated reports of a widespread lower motor neurone syndrome. Such bizarre anomalies, which may occur in other chronic meningitides, are one of the justifications for checking the CSF in patients being investigated for suspected motor neurone disease. Other causes of fungal meningitis are dependent on geography-for example, coccidiomycosis in the south western USA, Central and South America-underlining the need to obtain a detailed travel history in patients presenting with atypical meningitis.

\section{PROTOZOA AND METAZOA}

A disease indistinguishable from acute bacterial meningitis results from infection with the parasite Naegleria fowleri. The clue that this is the cause is a history of exposure. Naegleria is found in warm, stagnant water and soil, and patients, typically boys or young adult males, are infected by swimming in, or playing near, such areas. No organisms are seen on Gram stain, but motile trophozoites may be observed on direct microscopy of a fresh, warm CSF smear. Another protozoon, Acanthamoeba, causes a more chronic granulomatous meningoencephalitis which is very difficult to diagnose in life.

The rat lungworm, Angiostrongylus cantonensis, causes an eosinophilic meningitis in various parts of the world, originally Pacific regions, but now spread to Africa, India, the Far East, and elsewhere. Human infection is via contaminated food. Despite the unpleasant thought of live worms infesting the brain, most patients recover completely without specific treatment. Eosinophilic meningitis may also rarely be caused by another nematode, Gnathostoma, or cysticercosis.

\section{NON-INFECTIVE CAUSES}

The non-infective causes of aseptic meningitis are listed in table 1. Those likely to recur are described in more detail below. If the CSF glucose concentration is very low, then the cause is more likely to be malignant (carcinoma, lymphoma, leukaemia) than TBM for patients in the UK who are not in an at-risk group for tuberculosis. Particular difficulties may arise in differentiating sarcoidosis from tuberculosis and patients may need to be treated for both. The clinical context, however, can be helpful (see case study 2).
Table 2 Causes of viral meningitis

- Enteroviruses (echo, polio, coxsackie)

- Arboviruses*

- Herpes simplex virus (usually type 2)

- Lymphocytic choriomeningitis

- Varicella zoster, Epstein-Barr virus, cytomegalovirus

- Mumps

- Adenovirus

- Human immunodeficiency virus

*Numerous examples, dependent on geography, of which West Nile virus is currently the most newsworthy.

\section{RECURRENT MENINGITIS}

Recurrent meningococcal meningitis is associated with immune deficient states, notably defects of the complement system and agammaglobulinaemia. More usually, recurrent pyogenic meningitis implies an anatomical communication between the CSF space and the skin surface, or a non-sterile body cavity. Such lesions may be congenital or acquired. Congenital defects typically involve the skull base or middle ear, though persistent dermal sinuses may be found along the vertebral column, resulting from incomplete closure of the neural tube. Other rare congenital lesions include neurenteric fistulae and cysts. These anomalies generally present in childhood, but there are exceptions (see case study 3 ).

Acquired defects are seen most commonly in the context of head injury or neurosurgery. The classic lesion is a fracture of

\section{Case study 2}

- A 31 year old man of Afro-Caribbean origin presented with a subacute progressive history of unsteady gait and clumsy hands. Examination showed brisk tendon reflexes but downgoing plantar responses. Joint position sense was impaired in the hands and feet. A large intrinsic lesion of the upper cervical spinal cord, presumed inflammatory, was revealed on MR imaging. The CSF contained 11 lymphocytes/ $\mu$ with normal protein and glucose concentration. Serum angiotensin converting enzyme was grossly elevated and a gallium scan showed abnormalities typical of sarcoidosis. A diagnosis of probable neurosarcoidosis was made. He was treated with high dose corticosteroids, tapering to zero, and made a complete clinical and radiological recovery. Two years later, he developed a headache which became increasingly severe over two weeks. By that time, there was mild meningism but no focal neurological signs. His temperature was $37.5^{\circ} \mathrm{C}$. A CT cranial scan and subsequent MR imaging were normal. The CSF contained 150 white cells/ $\mu \mathrm{l}$, all lymphocytes, with normal protein and glucose concentration. No organisms were seen on Gram stain and there was no evidence of infection in the CSF on detailed investigation including PCR. His symptoms settled again, with a reducing course of steroids and no other treatment.

- Comment-This patient's antecedent history gave his doctors sufficient confidence to treat the episode of aseptic meningitis with steroids alone, despite the mild pyrexia. Without that history, management would have been more difficult, though the normal CSF glucose concentration is reassuring. Normal CSF glucose is by no means the rule for sarcoidosis. It is not uncommon for a patient to be treated simultaneously for neurosarcoidosis and TBM, at least until the results of $M$ tuberculosis cultures are known, sometimes for the full course of anti-TBM treatment, depending on the index of clinical suspicion. 


\section{Case study 3}

- A 39 year old woman presented with fulminant pyogenic meningitis postoperatively following surgery for haemorrhoids. At lumbar puncture, highly purulent CSF was obtained, containing multiple gut organisms. The patient succumbed to the overwhelming infection. At necropsy, a congenital sinus was identified, tracking from her natal cleft to the spinal canal.

- Comment-It is remarkable that this patient's first land only) presentation with meningitis was delayed until adulthood, in view of the finding of a congenital defect at a vulnerable site for bacterial ingress.

the delicate cribriform plate of the ethmoid bone. Very often the trauma responsible for a skull base defect is relatively minor and occurs years before the first episode of meningitis, by which time it may have been forgotten. The presence of CSF rhinorrhoea or otorrhoea is, of course, a valuable clue. Their absence, however, does not eliminate a communication between the CSF space and the nasal cavity or middle ear as the cause of recurrent pyogenic meningitis. Conversely, clear fluid discharge from the nose can be misleading. Proof that it is CSF has rested for many years on the presence of glucose but $\beta$-2 (tau) transferrin is a more specific CSF marker. Even in the presence of CSF rhinorrhoea, exact localisation of a CSF leak (as a prelude to surgical closure) is often difficult. Previous cumbersome methods involving instillation of isotope into the lumbar CSF and tracing its path by scintigraphy largely have been superseded by high definition CT and MR imaging, if necessary CT cisternography (fig 1 ).

In general, the management of recurrent pyogenic meningitis is to give standard treatment for the acute episode with appropriate antibiotics and supportive measures, and then if possible correct the underlying immunological or anatomical defect. The latter may require surgical closure but some

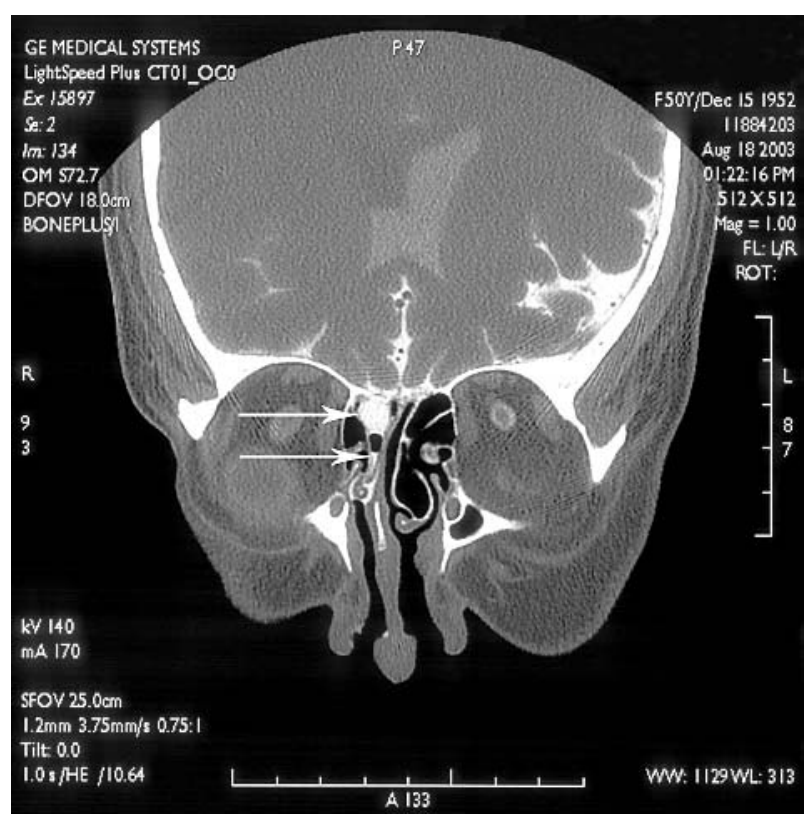

Figure $1 \mathrm{CT}$ cisternogram (coronal re-format) showing leakage of intrathecally introduced contrast medium into the ethmoidal air cells on the right (arrowed) in a 50 year old woman presenting with CSF rhinorrhoea and recurrent pyogenic meningitis.
Table 3 Drugs associated with a risk of recurrent aseptic meningitis

- Intrathecal agents (contrast medium, chemotherapy)

- Non-steroidal anti-inflammatory drugs

- Antibiotics (trimethoprim, amoxicillin, cephalosporins, etc)

- Cytotoxic and immunosuppressant drugs (azathioprine, cytarabine)

- Immunoglobulin

- OKT3 monoclonal antibody

lesions respond to conservative measures (bed rest with head up and avoidance of straining and other manoeuvres likely to increase intracranial pressure; repeated lumbar puncturesfor example, post-operatively in neurosurgical patients).

The causes of recurrent aseptic meningitis are numerous. Many are given in table 1 , but this list can be bewildering when applied to the management of individual patients. It is better to group the causes as follows, to avoid missing pertinent investigations:

- Infection-Chronic infective meningitis may present with recurrent clinical episodes separated by asymptomatic periods, even though the CSF is abnormal throughout. This pattern may be seen with syphilis, brucellosis, Lyme disease, fungal meningitis, and human immunodeficiency virus (HIV). With other organisms, a different mechanism of genuine reactivation of latent infection applies. The prime examples here are herpes simplex virus (HSV), Epstein-Barr virus (EBV), and Toxoplasma.

- Structural lesions-Recurrent chemical meningitis occurs when structural lesions along the neuraxis periodically discharge their contents into the CSF. This phenomenon is well known for craniopharyngiomas, epidermoid cysts, and gliomas, but other causes have been described.

- Drugs-Many drugs are associated with a risk of recurrent aseptic meningitis (table 3). This list is far from exhaustive-isolated case reports have implicated numerous other agents.

- Chronic inflammatory diseases (and other non-infective causes) Recurrent aseptic meningitis is a recognised feature of sarcoidosis, lupus, and other connective tissue disorders, vasculitis, Behçet's disease, and recurrent hereditary polyserositis. Vogt-Koyanagi-Harada syndrome is a very rare condition in which aseptic meningitis is associated with sensorineural deafness, uveitis, retinal haemorrhages, and skin and hair depigmentation. Migraine may rarely present with fever, symptoms suggestive of meningo-encephalitis, and CSF pleocytosis, but this must be a diagnosis of exclusion.

The syndrome of benign recurrent aseptic meningitis of unknown cause is called Mollaret's meningitis. Patients typically experience episodes of fever and meningism of abrupt onset lasting 3-4 days. Occasionally, there are seizures and focal neurological signs. They then return to normal for weeks, months or years until the next episode. Usually, after several attacks, the condition seems to "burn out" within a few years. Examination of the CSF during an episode shows a pleocytosis, sometimes several thousand cells per microlitre, with lymphocytes, neutrophils and so-called "Mollaret cells"-large, friable cells with faintly staining vacuolated cytoplasm. These cells originally were thought to be endothelial but are now considered to be activated macrophages and are not pathognomonic of the disorder. Whether the term "Mollaret's meningitis" will survive the discovery of an association with HSV (usually HSV-2) in many of these patients ${ }^{10}$ is both a semantic and a practical issue. The role of 
acyclovir in the prevention or treatment of exacerbations is yet to be firmly established.

\section{ACKNOWLEDGEMENTS}

I thank Dr Lloyd Savy, consultant neuroradiologist, for permission to reproduce fig 1 .

\section{REFERENCES}

1 Kavanagh S. Diagnosing acute headache: avoiding pitfalls - a guide to practice. MPS Casebook 2003;UK No. 3:8-14.

"But for the grace of God there goes ..."

2 Davenport R. Acute headache in the emergency department. J Neurol Neurosurg Psychiatry 2002;72(suppl II):ii33-7.

- A clear summary of the differential diagnosis of acute headache.

3 Hasbun R, Abrahams J, Jekel J, et al. Computed tomography of the head before lumbar puncture in adults with suspected meningitis. N Engl J Med $2001 ; 345: 1727-33$

- This study confirms that only a small proportion of patients with suspected meningitis are at risk of cerebral herniation after lumbar puncture.

4 Quagliarello VJ, Scheld WM. Treatment of bacterial meningitis. N Engl J Med 1997;336:708-16.

- A definitive account.
5 Ginsberg L, Kennedy DH. Meningitis, bacterial. In: Pounder R, Hamilton M, eds. Handbook of current diagnosis and treatment. Edinburgh: Churchill Livingstone, 1995.

- A brief summary of the diagnosis and management of pyogenic meningitis.

6 de Gans J, van de Beek D, for the European Dexamethasone in Adulthood Bacterial Meningitis Study Investigators. Dexamethasone in adults with bacterial meningitis. N Engl J Med 2002;347:1549-56.

- Evidence of the beneficial effect of early treatment with corticosteroids.

7 Kastenbauer S, Pfister H-W. Pneumococcal meningitis in adults. Brain 2003;126:1015-25.

- Complications and prognosis in a large series of patients.

8 Thwaites GE, Chau TT, Stepniewska K, et al. Diagnosis of adult tuberculous meningitis by use of clinical and laboratory features. Lancet 2002;360:1287-92.

- A diagnostic rule for use particularly in settings with limited microbiological resources.

9 Prasad K, Volmink J, Menon GR. Steroids for treating tuberculous meningitis. Cochrane Database 2000;Syst Rev:3.

A meta-analysis of six randomised trials.

10 Tedder DG, Ashley R, Tyler KL, et al. Herpes simplex virus infection as a cause of benign recurrent lymphocytic meningitis. Ann Intern Med 1994; 121:334-8

- Evidence that Mollaret's meningitis is associated with herpes simplex virus (mainly HSV-2). 